Rev. Saúde públ., s. Paulo

7:241-50, 1973 .

\title{
MEDIDA DA INFESTAÇÃO DOMICILIAR POR TRIATOMA SORDIDA *
}

\author{
Oswaldo Paulo FORATTINI ** \\ Octávio Alves FERREIRA *** \\ José Maria Pacheco de SOUZA ** \\ Ernesto Xavier RABELLO ** \\ Eduardo Olavo da ROCHA E SILVA *** \\ Flavio Wagner RODRIGUES *****
}

\begin{abstract}
RSPU-B/175
Forattini, O. P. et al. - Medida da infestação domiciliar por Triatoma sordida. Rev. Saúde públ., S. Paulo, 7: 241-50, 1973.

Resumo: Procurou-se verificar a viabilidade do uso de abrigos artificiais de madeira tipo "calha" para diagnóstico de infestação domiciliar por Triatoma sordida. Foi observada uma amostra de 45 casas e 27 anexos, em duas localidades pertencentes ao municpio de Guaíra na regiäo Norte do Estado de São Paulo (Brasil), durante um período de 7 meses, a partir de outubro de 1972. Os resultados foram considerados favoráveis ao uso dos abrigos nos anexos e desfavoráveis ao uso nas casas. Apresentam-se possiveis razões para esta uiltima situação.
\end{abstract}

UNITERMos: Tripanossomose americana*; Triatomineos domiciliares (medida de infestação) *; Triatoma sordida *.

\section{N TROD U CAO}

O uso de abrigos artificiais, com o objetivo de investigar a presença de triatomíneos em casas e anexos, foi preconizado para algumas espécies, notadamente Rhodnius prolixus na Venezuela e Triatoma infestans no Estado de São Paulo, Brasil. Para tanto, tem-se experimentado o emprego de caixas de papelão de tamanho padronizado, e que permitissem fácil exame periódico (GÓMEZ-NÚNEZ ${ }^{3}$, 1965; GAMBOA 4 , 1965; Forattini et al. 5, 6, 1969). Os resultados foram bastante animadores, face à simplicidade do método, permitindo assim a sua recomendação como um dos processos de trabalho a serem utilizados para áreas em vigilância (RochA e SiLva et al. 11, 12, 1969, 1970; Forattini et al. 7, 1971).

* Realizado com o auxilio do "U.S. Army Research Office for South America" (Convênios DAHC 19-70-G-0021 e DAHC 19-71-G-0015).

* Do Departamento de Epidemiologia da Faculdade de Saúde Pública da USP - Av. Dr. Arnaldo, 715 - São Paulo, SP - Brasil.

* * Da Diretoria de Combate a Vetores da Superitendência do Saneamento Ambiental (SUSAM) do Estado de São Paulo - Rua Tamandaré, 649 - Sáo Paulo, SP - Brasil.

**** Do Instituto de Matemática e Estatística da USP - Cidade Universitária - São Paulo, SP - Brasil. 
FORATTINI, O. P. et al. - Medida da infestação domiciliar por Triatoma sordida. Rev. Saúde públ., S. Paulo, 7:241-50, 1973.

Os recentes estudos dos assim chamados triatomíneos semidomésticos no Estado de São Paulo, tem revelado a capacidade invasiva do Triatoma sordida em relação aos ambientes domiciliares e peridomiciliares, a partir de seus ecótopos naturais (Foratrini et al. 8,9 1971). Além disso, trata-se de espécie que não tem respondido de maneira inteiramente satisfatória aos métodos de controle utilizados (Rocha e Sirva et al.11, 1969). Haveria pois, apreciável interesse em verificar a eficiência do uso de abrigos artificiais na detecção de infestações domiciliares por parte desse inseto. Tendo em vista tal objetivo, foram levadas a efeito observações nas localidades denominadas Bairro João Pedro e Fazenda Bananal, no município de Guaíra, situado na região norte do Estado de São Paulo. As características gerais dessa região já foram objeto de considerações em trabalho anteriormente publicado (Forattini et al. 8, 1971).

\section{MATERIAL E METODOS}

Os resultados conseguidos com o emprego de esconderijos em ecótopos naturais, ensejou a possibilidade de verificar a utilização desses artefatos em ambiente domiciliar (ForatTinI et al.10, 1973). Sua construção foi feita utilizando-se duas tábuas de madeira, de $40 \mathrm{~cm}$ de comprimento e $10 \mathrm{~cm}$ de largura, unidas de maneira a formarem pequena "calha". Em seu interior foi disposta folha de papel dobrada em sanfona, com - objetivo de servir de suporte aos triatomíneos. Assim constituídos, os abrigos foram dependurados com a face voltada para a parede, em locais previamente escolhidos (Figuras 1 a 4 ).

Em outubro de 1972, foi feita uma inspeção preliminar em todas as 98 casas e respectivos 78 anexos da regiáo, obtendo-se informações sobre positivi-

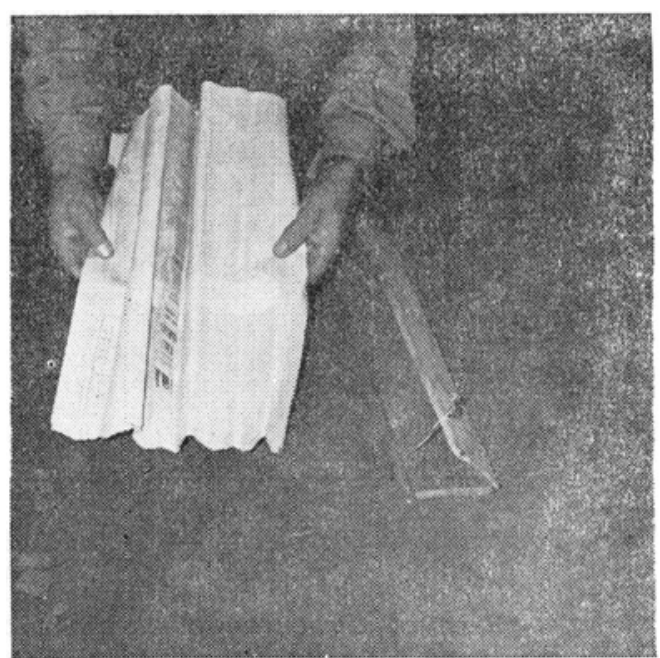

Fig. 1 - Aspecto do esconderijo tipo "calha".

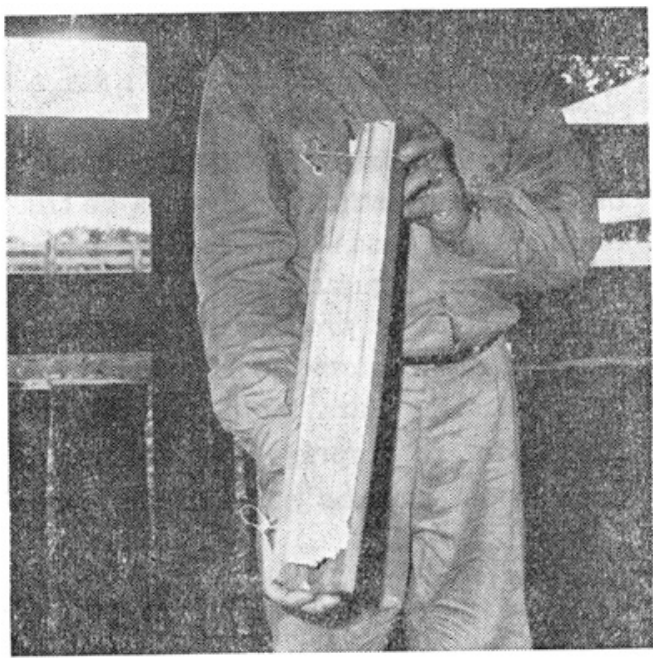

Fig. 2 - $O$ mesmo artefato da figura anterior, mostrando sua construção e a folha de papel dobrada, em seu interior. 
FORATTINI, O. P. et al. - Medida da infestação domiciliar por Triatoma sordida. Rev. Saúde puibl., S. Paulo, 7:241-50, 1973.

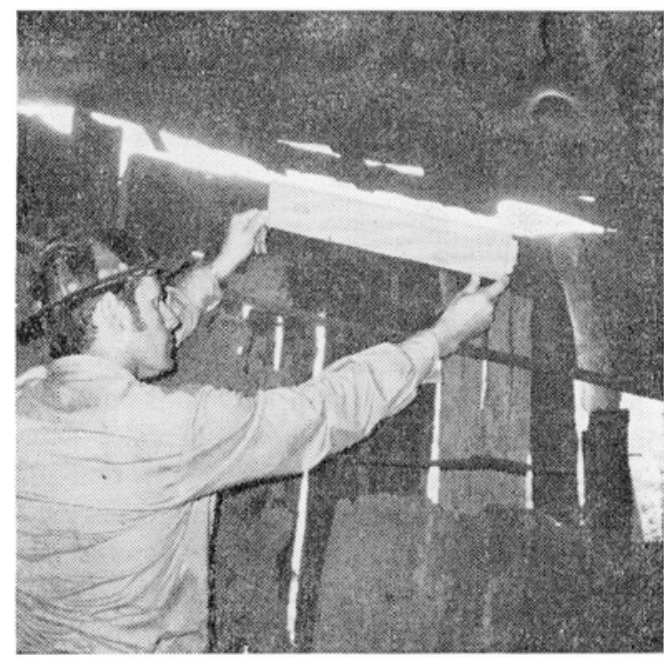

Fig. 3

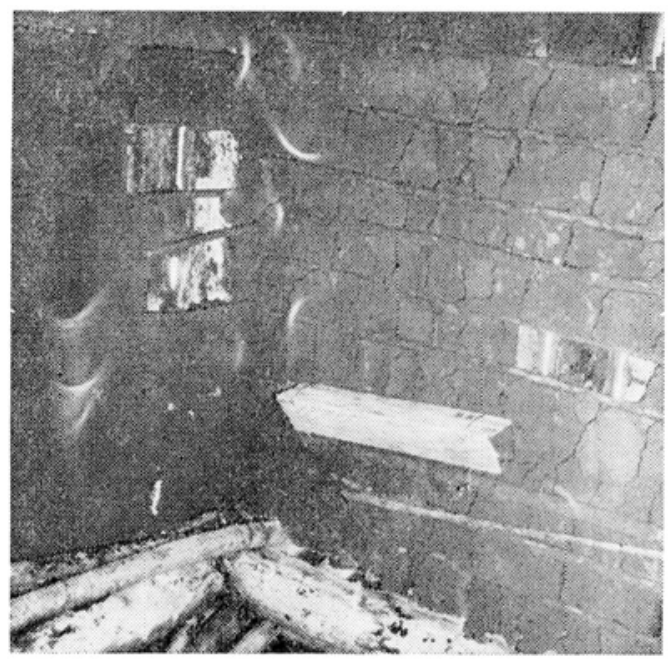

Figs. 3 e 4 - Colocação dos esconderijos em anexos e domicílios.

dade de cada edifício. A partir das informações obtidas neste exame prévio, decidiu-se incluir no estudo:

a) todas as casas infestadas, com ou sem anexos e, em caso de possuí-los, infestados ou não. (posteriormente não foi possível localizar um desses domicílios);

b) um número igual de casas não infestadas, com anexo não infestado ou sem anexo (obtidas por sorteio);

c) todas as restantes casas não infestadas mas com anexos infestados.

A Tabela 1 apresenta a distribuição de casas e anexos na região e aquela obtida para o estudo.

T A B E L A 1

Distribuição das casas e anexos segundo positividades, no total e na amostra.

\begin{tabular}{c|c|c|c}
\hline Casa & Anexo & Total & Amostra \\
\hline+ & + & 9 & 9 \\
+ & - & 7 & 7 \\
+ & $\theta$ & 2 & 1 \\
- & - & 51 & 12 \\
- & 0 & 18 & 5 \\
- & + & 11 & 11 \\
& & 98 casas & $\begin{array}{c}45 \text { casas } \\
\text { Totais }\end{array}$ \\
\hline
\end{tabular}

+ apresentou infestaçăo

- não apresentou infestação

$\theta$ não existe anexo

(a) dos quais 12 não receberam abrigos.

Em cada casa foram colocados 2 abrigos, um junto à cama do casal e outro na cozinha. Em cada anexo positivo, ou negativo com casa positiva, foi também pendurado um abrigo. Não se estudaram anexos negativos pertencentes a casas negativas. A cada 14 dias após a instalação das "calhas" (novembro, 1972), procedia-se à inspeção, que foram em número de 10. Em maio de 1973 foi 
FORATTINI, O. P. et al. - Medida da infestação domiciliar por Triatoma sordida. Rev. Saúde puibl., S. Paulo, 7:241-50, 1973.

feito um exame manual, semelhante ao preliminar, compreendendo apenas as casas e anexos da amostra, encerrando-se, então, a fase de levantamento de dados.

Algumas casas e anexos tiveram que ser abandonados pelo estudo, principalmente devido a demolições.

E importante ter-se em conta que todo triatomíneo encontrado era recolhido e enviado a São Paulo para exame. Isto implica que casas, anexos ou abrigos achados infestados mais de uma vez, o eram por diferentes espécimens. A razão do reexame final foi justamente a de controlar o efeito da retirada dos insetos infestantes.

Para análise estatística usou-se o teste Q, a um nível de significância de $5 \%$ (Cochran 1 , 1950; Cochran \& Cox 2,1966 ).

\section{RESULTADOS E COMENTARIOS}

Na Tabela 2 são encontrados todos os resultados da amostra. Pôde-se, então, obter a estimativa de infestação domiciliar para aquela região, válida para a ocasião da primeira inspeção. Em 98 casas, 17 foram achadas positivas, ou seja, 17,35\%. Para os anexos, tem-se 20 positivos em 78 , isto é, $25,64 \%$. Deve-se chamar a atenção para o fato de que como anexo, subentende-se um conjunto de construções externas pertencentes a uma mesma casa, fato este que pode conduzir a diferentes interpretações. A Tabela 2 apresenta alguns dados complementares que se decidiu não utilizar neste trabalho.

As Tabelas 3 a 12 informam qual o comportamento da positividade, segundo os três tipos de inspeção, para as casas e para os anexos. Um possivel resultađo favorável ao futuro uso de abrigos de madeira, seria aquele em que, tendo o exame preliminar acusado infes- tação, as inspeções usando abrigos a confirmassem; para exame inicial negativo, os exames de abrigo poderiam ser também negativos, ou positivos, indicando, então, uma infestação nova ou falha no exame inicial.

Pela Tabela 3, que se refere às 40 casas que puderam ser examinadas em todas as ocasiōes, vê-se um comportamento nitidamente diferente quanto aos achados positivos para exame inicial e para exames dos abrigos e final. Em $25 \%$ das vezes ( 10 casas em 40 ) tem-se exame inicial positivo e os seguintes negativos. Apenas em $\mathbf{5 \%}$ das vezes (2 casas em 40) houve concordância total. O teste $Q$ de Cochran 1 (1950) acusou o valor de 14,80 que, comparado com o valor crítico da distribuição $X^{2}$ com 2 graus de liberdade $(5,991)$, indica de fato, que a hipótese de diferença de positividade nas 3 ocasiões pode ser aceita. Fazendo-se a partição do $\mathrm{X}^{2}$ resultante (Cochran \& Cox ${ }^{2}, 1966$ ), obteve-se $Q_{1}=14,70$, com um grau de liberdade, para a diferença entre positividade no exame final e média das positividades nos exames dos abrigos e final; aceita-se então a hipótese de comportamento diferente entre exame inicial e os outros dois.

Nas Tabelas 4 a 7, tem-se uma apresentação mais pormenorizada da Tabela 3 , com as comparações de ocasião de exame aos pares. Também tem-se uma comparação entre exame inicial e o conjunto de exames de abrigos e final, onde se considerou resultado positivo sempre que qualquer destas duas inspeções fosse positiva. As Tabelas 4 e 5 mostram resultados bastante semelhantes; concordâncias quanto a resultados negativo/negativo são da ordem de 60\% (24 em 40) em ambas; para resultados positivo/positivo tem-se somente $7,5 \%$ e $10 \%$ ( 3 e 4 em 40). Restam então os pares discordantes, 13 e 12, respectivamente. 
T A B E L A 2

Resultados obtidos em casas e anexos no bairro João Pedro (JP) e na fazenda Bananal (BA), de infestação por Triatoma sordida, segundo inspeção preliminar, uso de abrigos de madeira e inspeção final.

\begin{tabular}{|c|c|c|c|c|c|c|c|}
\hline \multirow{2}{*}{$\begin{array}{c}\text { Identificação e } \\
\text { de construção } \\
\text { casa }\end{array}$} & \multirow{2}{*}{$\begin{array}{l}\text { tipo } \\
\text { da }\end{array}$} & \multicolumn{2}{|c|}{$\begin{array}{l}\text { Inspeção } \\
\text { preliminar }\end{array}$} & \multicolumn{2}{|c|}{$\begin{array}{c}\text { Abrigos de } \\
\text { Madeira }\end{array}$} & \multicolumn{2}{|c|}{$\begin{array}{l}\text { Inspeção } \\
\text { final }\end{array}$} \\
\hline & & Casa & Anexo & Casa & Anexo & Casa & Anexo \\
\hline $1-\mathrm{JP1}$ & $\mathbf{r}$ & - & $\varnothing$ & - & $\varnothing$ & - & $\varnothing$ \\
\hline $2-\mathrm{JP2}$ & $\mathbf{r}$ & + NO & - & - & $=$ & $\mathbf{P}$ & $\widehat{\mathbf{P}}$ \\
\hline $3-J P 16 A$ & $\mathbf{b}$ & - & $\varnothing$ & - & $\varnothing$ & - & $\varnothing$ \\
\hline $4-J P 17$ & $\mathbf{r}$ & - & $\simeq$ & - & $\tilde{\theta}$ & - & $\simeq$ \\
\hline $5-J P 21$ & $\mathbf{b}$ & $+\mathbf{N}$ & $+N$ & $+\mathrm{N} 8(\mathrm{a})$ & $+\mathrm{N} 3$ & $+\mathbf{N A}$ & $+N$ \\
\hline $6-J P 21 A$ & $\mathbf{b}$ & $+N$ & $+N$ & $+\mathbf{N 1}$ & $\mathbf{P}$ & - & - \\
\hline $7-J P 22$ & $\mathbf{r}$ & $+\mathbf{N}$ & - & - & $+N 1$ & - & - \\
\hline $8-J P 23$ & $\mathbf{r}$ & - & - & - & $\theta$ & - & - \\
\hline $9-$ JP25 & $\mathrm{m}$ & $+N$ & - & - & $+\mathbf{N 1}$ & - & - \\
\hline $10-J F 26$ & $\mathbf{b}$ & - & $+N$ & - & - & - & $+\mathbf{N A}$ \\
\hline $11-J P 28$ & $\mathbf{b}$ & - & $+\mathbf{N}$ & - & - & - & - \\
\hline $12-J P 30$ & $\mathbf{b}$ & - & - & - & - & $\mathbf{P}$ & $\mathbf{P}$ \\
\hline $13-J P 37$ & $\mathbf{r}$ & - & - & - & $\theta$ & - & 一 \\
\hline $14-\mathrm{JP} 39$ & $\mathbf{b}$ & -- & - & - & 0 & - & - \\
\hline $15-J P 43$ & $\mathbf{r}$ & $+\mathrm{NA}$ & $+\mathbf{N A}$ & +02 & $+N 8$ & - & $+\mathbf{A}$ \\
\hline $16-J P 45$ & $\mathbf{b}$ & - & $\varnothing$ & - & $\varnothing$ & - & $\varnothing$ \\
\hline $17-J P 45 A$ & $\mathbf{b}$ & - & - & $+\mathbf{A 9}$ & $\theta$ & - & - \\
\hline $18-J P 45 B$ & $\mathbf{b}$ & - & - & - & $\theta$ & - & - \\
\hline $19-J P 48$ & $\mathbf{r}$ & - & $+\mathbf{N}$ & - & - & - & $+\mathbf{A}$ \\
\hline $20-J P 50$ & $\mathbf{r}$ & +NAO & - & - & $+\mathrm{N} 2$ & - & - \\
\hline $21-J P 50 A$ & $t$ & $+N$ & - & - & $\mathbf{P}$ & - & $\mathbf{P}$ \\
\hline $22-J P 51$ & b & - & $+N$ & - & - & - & - \\
\hline $23-\mathrm{JP52}$ & $\mathbf{b}$ & $+\mathbf{A}$ & $\cdots$ & - & $+N 1$ & - & - \\
\hline $24-J P 52 A$ & $\mathbf{r}$ & - & $+N$ & - & - & - & $+\mathbf{N}$ \\
\hline $25-J P 54$ & b & - & - & - & 0 & - & $+\mathbf{N}$ \\
\hline $26-J P 54 A$ & $\mathbf{I}$ & - & - & - & 0 & - & - \\
\hline $27-J P 54 B$ & b & $+N$ & - & - & - & - & - \\
\hline $28-J P 54 C$ & $\mathbf{b}$ & $+N$ & $\varnothing$ & - & $\varnothing$ & - & $\varnothing$ \\
\hline $29-J P 54 D$ & $\mathbf{m}^{-}$ & $+N$ & $+N$ & $+N 1$ & $\underline{-}$ & $\mathbf{P}$ & $\widetilde{\mathbf{P}}$ \\
\hline $30-J P 56$ & $\mathrm{~m}$ & $+N$ & $+N$ & - & $+N 7$ & $+\mathbf{N A}$ & - \\
\hline $31-J P 57$ & b & $+\mathrm{N}$ & $+N$ & + NAl & - & $+A$ & $+\mathbf{A}$ \\
\hline $32-J P 57 B$ & b & - & $+\mathbf{N}$ & - & +NA2 & $+\mathbf{N}$ & $+\mathbf{N}$ \\
\hline $33-B A 5$ & $\mathbf{b}$ & $+\mathrm{A}$ & $+\mathbf{A}$ & - & $+\mathrm{A} 3$ & - & $+\mathbf{N}$ \\
\hline $34-B A 22 A$ & $\mathbf{r}$ & - & $+\mathrm{N}$ & - & - & - & - \\
\hline $35-$ BA22B & $\mathrm{b}$ & - & $m$ & - & 0 & - & - \\
\hline $36-$ BA24B & $\mathbf{r}$ & $+N$ & $+N$ & - & - & - & $+\mathbf{N}$ \\
\hline $37-\mathbf{B A 2 5}$ & $\mathbf{r}$ & - & $+\mathbf{N}$ & - & - & - & - \\
\hline 38 - BA28 & $\mathbf{t}$ & - & $\varnothing$ & - & $\varnothing$ & - & $\varnothing$ \\
\hline $39-\mathrm{BA} 31$ & $\mathbf{m}$ & $+N$ & $T+N$ & - & $+\mathrm{N} 1$ & - & - \\
\hline $40-B A 35$ & $t$ & - & $+N$ & - & $+N 1$ & - & $+\mathbf{A}$ \\
\hline $41-\mathrm{BA37}$ & $\mathbf{r}$ & - & - & - & $\theta$ & - & - \\
\hline $42-\mathrm{BA} 43$ & $\mathbf{r}$ & - & $+N$ & - & - & - & $+\mathbf{N}$ \\
\hline $43-\mathrm{BA} 53$ & b & - & $\varnothing$ & $\mathbf{p}$ & $\mathbf{P}$ & $\mathbf{P}$ & $\mathbf{P}$ \\
\hline $44-$ BA56 & $\mathbf{r}$ & - & $+N$ & - & $+\mathrm{N} 7$ & - & $+N A$ \\
\hline $45-B A 56 F$ & $\mathbf{t}$ & - & - & $\mathbf{P}$ & $\mathbf{P}$ & $\mathbf{P}$ & $\mathbf{P}$ \\
\hline
\end{tabular}

(a): o número após o sinal + refere-se à inspeção em que foi verificada a positividade pela primeira vez.
$\mathbf{r}$ - tijolo revestido
+ : casa ou anexo infestado
N : ninfa
t - tijolo nu
b - barro
- : casa ou anexo não infestado
$\varnothing:$ anexo inexistente
A : adulto
$\mathrm{m}$ - madeira
$\theta$ : anexo não examinado
O: ôvo
$\stackrel{\theta}{P}$ : perdido de observação 
FORATTINI, O. P. et al. - Medida da infestaçāo domiciliar por Triatoma sordida. Rev. Saúde públ., s. Paulo, 7:241-50, 1973.

A partir destes pares discordantes infere-se que há grande desvantagem, quanto a diagnóstico de positividade, para os exames posteriores ao inicial. Usando-se a Tabela 4, tem-se que 12 casas infestadas segundo o exame preliminar não foram confirmados pela inspeção final, havendo somente uma casa na condição recíproca. Na Tabela 5, a situação é praticamente a mesma, tendo-se 11 casas negativas pela inspeção dos abrigos que eram positivos no início, e uma casa em situação contrária.

A Tabela 6 dá indicações de semelhança de resultados entre abrigos e inspeção final. E a Tabela 7 confirma a deficiência de resultados positivos para exames posteriores ao inicial; pode-se inclusive calcular um índice de especificidade, que é da ordem de apenas $33,33 \%$.

$$
\text { T A B E L A } 3
$$

Número de casas segundo as possíveis combinações de diagnósticos de infestação das três inspeçóes (iniclal, dos abrigos e final)

\begin{tabular}{c|c}
\hline Diagnóstico & N.0 \\
\hline+++ & 2 \\
++- & 2 \\
+-+ & 1 \\
+-- & 10 \\
-++ & 0 \\
--+ & 1 \\
-+- & 1 \\
-- & 23 \\
\hline Total & 40 \\
\hline
\end{tabular}

T A B E L A 4

Diagnósticos de infestação das casas, segundo inspeções inicial e final.

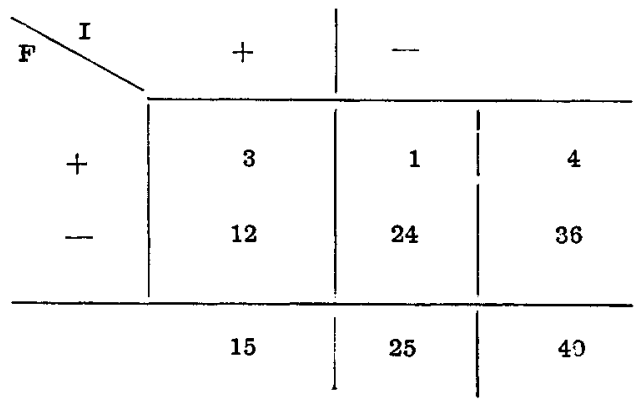

T A B E L A D

Diagnósticos de infestaçōes das casas, segundo inspeção inicial e dos abrigos.

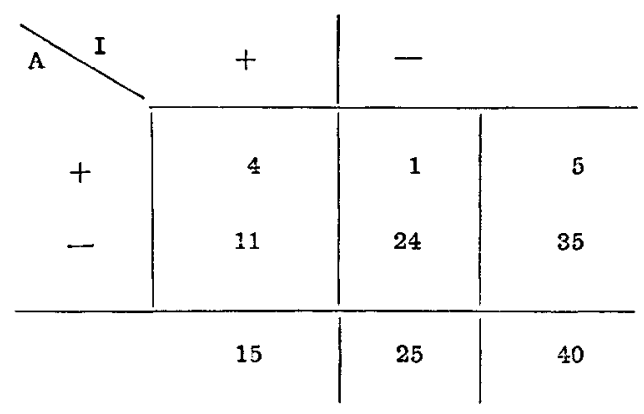

T A B E L A 6

Diagnósticos de infestaçōes das casas, segundo inspeção dos abrigos e final.

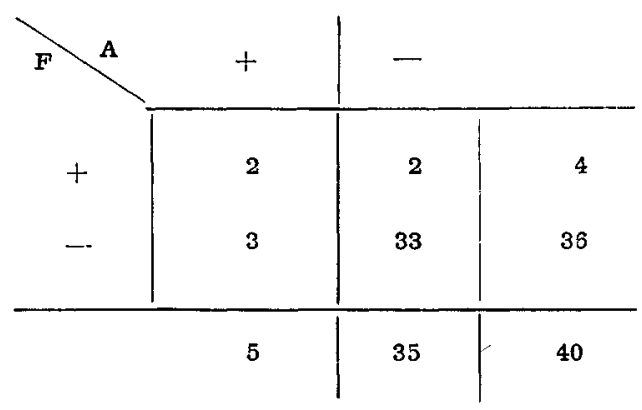


FORATTINI, O. P. et al. - Medida da infestação domiciliar por Triatoma sordida. Rev. Saúde públ., S. Paulo, 7:241-50, 1973.

\section{T A B E L A 7}

Diagnósticos de infestações das casas, segundo inspeçăo inicial e resultado combinado das inspeções de abrigos e final.

\begin{tabular}{l|l|l|l}
\multicolumn{1}{c|}{\begin{tabular}{l|l} 
Combi- \\
nado \\
+
\end{tabular}} & 5 & 2 & \multicolumn{2}{l}{} \\
\cline { 2 - 4 }- & 10 & 23 & 33 \\
\hline & 15 & 25 & 40
\end{tabular}

Com base nestas observações parece lícito supor-se que a retirada do inseto ou insetos que determinou ou determinaram o diagnóstico de positividade inicial, causa um prejuizo às futuras observações, distorcendo a avaliação da efetividade dos abrigos. Não sendo possível deixar o triatomíneo na casa, por motivos éticos, esta avaliação terá que ser feita por métodos indiretos. Acrescente-se a característica conhecida do pequeno número de vestígios produzidos pelo Triatoma sordida. Isso, pois, redunda em fator de menor positividade na leitura dos abrigos quando comparada com a de outros triatomíneos como - Rhodnius prolixus na Venezuela e Triatoma infestans no Brasil.

As Tabelas 8 a 12 são analisadas segundo a mesma linha, sendo relativas, porém, aos achados dos anexos. Observa-se que o fenômeno é diferente quando comparado com o acontecido nas casas.

Calculando-se a estatística $Q$ para a Tabela 8, tem-se o valor 4,5 que é não significante, indicando que as inspeçōes iniciais, de abrigos e final não diferem entre si quanto à proporção de positividade. As Tabelas 9 e 10 mostram que, comparando-se a inspeção de abrigos e a inspeção final há uma leve vantagem para a primeira, se se toma a inspeção preliminar como padrão, vantagem esta que não é mostrada pela Tabela 11.

A Tabela 12, onde os achados da inspeção de abrigo e final são considerados em conjunto, simplesmente confirma o resultado estatístico verificado $\mathrm{na} T \mathrm{Ta}-$ bela 8. $O$ indice de sensitividade foi da ordem de $\mathbf{7 7 , 7 8} \%$.

Portanto, para os anexos, é de se supor que os abrigos de madeira atuem de ma-

$$
\text { T A B E L A } 8
$$

Número de anexos segundo as possíveis combínações de diagnósticos de infestação das três inspeções (inicial, dos abrigos e final).

\begin{tabular}{c|c}
\hline Diagnóstico & N.0 \\
\hline+++ & 6 \\
++- & 2 \\
+-+ & 6 \\
+-- & 4 \\
-++ & 0 \\
--+ & 0 \\
-+- & 4 \\
-- & 1 \\
\hline
\end{tabular}

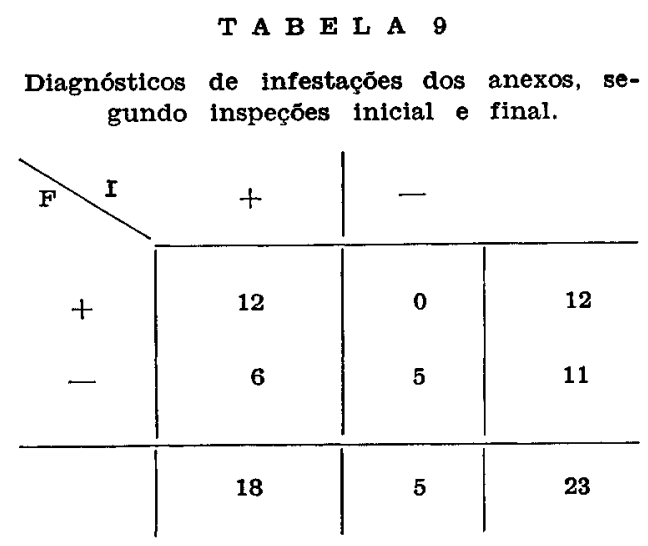


FORATTINI, O. P. et al. - Medida da infestação domiciliar por Triatoma sordida. Rev. Saúde públ., S. Paulo, 7:241-50, 1973.

T A B E L A 10

Diagnósticos de infestaçóes dos anexos, segundo inspeções inicial e dos abrigos.

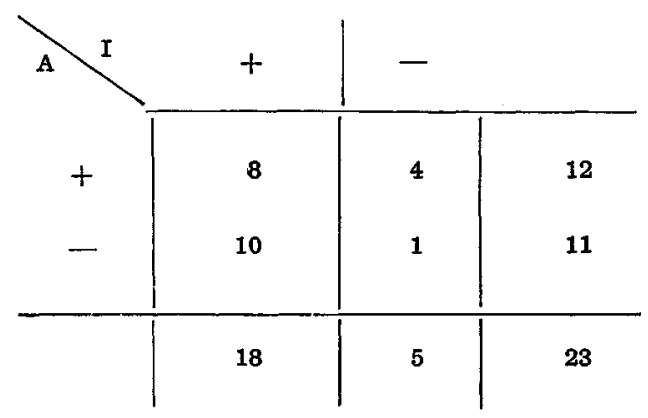

T A B E L A 11

Diagnósticos de infestaçóes dos anexos, segundo inspeçóes dos abrigos e final.

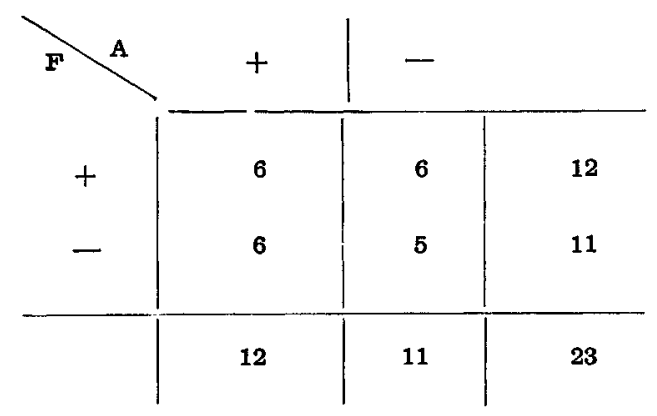

T A B E L A 12

Diagnósticos de infestações dos anexos, segundo inspeção inicial e resultado combinado das inspeçōes de abrigos e final.

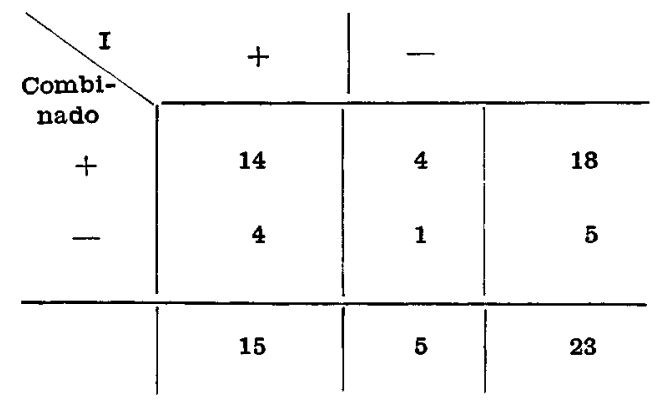

neira satisfatória. Algumas hipóteses podem ser aventadas para este comportamento diferente daquele das casas:

a) a inspeção manual do anexo é mais trabalhosa, talvez dificultando as capturas;

b) apenas o anexo mais próximo à casa foi usado para colocação dos abrigos de madeira, enquanto que, para os exames preliminar e final, devido a critério de rotina do serviço de profilaxia encarregado das inspeções, todo o conjunto de anexos de cada casa foi considerado;

c) no estado atual, há indícios de que o Triatoma sordida esteja em fase de invasão e adaptação do ambiente humano. Nessa hipótese, seria de se esperar maior densidade do inseto nos anexos, os quais constituiriam fatores de atração mais acentuađa sobre a população silvestre.

\section{CONCIUSOES}

Parece lícito aceitar-se o uso de abrigos em anexos afim de detectar possível infestação por Triatoma sordida.

Para as casas, os resultados foram desfavoráveis, podendo-se atribuir esta situação possivelmente à captura e retirada prévia do inseto. A isso, provavelmente, acrescente-se a escassez de vestígios, própria desse triatomíneo, quando comparado com outras espécies.

Contornada a dificuldade de ordem ética, talvez se possam obter resultados mais conclusivos. 
FORATTINI, O. P. et al. - Medida da infestação domicillar por Triatoma sordida. Rev. Saude públ., s. Paulo, 7:241-50, 1973.

RSPU-B $/ 175$

Forattini, O. P. et al. - [Measurements of house infestation on by the Triatoma sordida.] Rev. Saúde públ., S. Paulo, 7: 241-50, 1973.

SUMmary: In order to know if it is possible the use of wooden boxes in diagnosing house infestation by Triatoma sordida, a sample of 45 houses and 27 annexes was observed during seven months, beginning in October 1972 , in two sites in the county of Guaira, in the Northern region of the State of S. Paulo (Brazil). The results were considered good for using the boxes in out buildings, but not so for the houses; some possible reasons for this last failure are presented.

UNiterms: Trypanosomiasis South American*; Triatomid, measure of houses infestation *; Triatoma sordida *.

\section{REFERENCIAS BIBLIOGRAFICAS}

1 - COCHRAN, W. G. - The comparison of percentagens in matched samples. Biometriks, 37:256-66, 1950.

2 - COCHRAN, W. G. \& COX, G. M. Experimental design. 2nd edition. New York, Wiley \& Sons, 1966. p.63.

3 - GÓMEZ-NƯNEZ, J. C. - Desarollo de un nuevo método para evaluar la infestación intradomiciliaria por Rhodnius prolixus. Acta cient. venez., 16:26-31, 1965.

4 - GAMBOA, C. J. - Comprobación de la presencia de Rhodnius prolixus en la vivienda rural. Bol. inf. Dir. Malar. San. amb., 5:270-4, 1965.

5 - FORATTINI, O. P. et al. - Medida da infestação domiciliar por Triatoma infestans. Rev. Saúde públ., S. Paulo, 3:11-16, jun. 1969.

6 - FORATTINI, O. P. et al. - Infestação domiciliar por Triatoma infestans e alguns aspectos epidemiológicos da tripanossomose americana em área do Estado de São Paulo, Brasil. Rev. Saúde públ., S. Paulo, 3:159-72, 1969.

7 - FORATT'INI, O. P. et al. - Observações sobre a infestação domiciliar residual por Triatoma infestans. Rev. Saúde públ., $\mathrm{S}$. Paulo, 5:17-21, 1971.

8 - Forattini, O. P. et al. - Aspectos ecológicos da tripanossomose americana. II - Distribuição e dispersão local de triatomíneos em ecótopos naturais e artificiais. Rev. Saúde públ., s. Paulo, 5:163-91, 1971.

9 - FORATtinI, O. P. et al. - Aspectos ecológicos da tripanossomose americana. III - Dispersão local de triatomíneos, com especial referência ao Triatoma sordida. Rev. Saúde puibl., S. Paulo, 5:193-205. 1971. 
FORATTINI, O. P. et al. - Medida da infestação domiciliar por Triatoma sordida. Rev. Saúde puibl., S. Paulo, 7:241-50, 1973.

10 - FORATTINI, O. P. et al. - Aspectos ecológicos da tripanossomose americana. V - Observações sobre colonização espontânea de triatomíneos silvestres em ecótopos artificiais, com especial referência ao Triatoma sordida - Rev. Saúde públ., S. Paulo, 7:219-39, 1973.

11 - ROCHA e SILVA, E. O. da et al. - Suspensão do rociado no combate ao
Triatoma infestans em áreas do Estado de São Paulo, Brasil. Rev. Saúde públ., S. Paulo, 3:173-81, 1969.

12 - ROCHA e SILVA, E. O. da et al. Doença de Chagas - Atividades de vigilância entomológica desenvolvida numa área do Estado de São Paulo, Brasil. Rev. Saude públ., S. Paulo, 4:129-45, 1970.

Recebido para publicação em 13-6-1973

Aprovado para publicação em 10-7-1973 